\title{
Computational Theory and Key Issues
}

\author{
Varanga Sarma
}

\begin{abstract}
The enormous prominence of computer and information technology at the commencement of the 21st century has sincerely valued an academic experience to acquire on the matter, which originally perceived all viewpoints of our social, technological, and experimental lives. The theory of computation is an innovative, significant and fascinating theory of mathematics, physics, and other frequently addressed themes. The purpose of the theory of computation evolution is to emerge formal mathematical principles of computation that indicate real-world machines. The theory of computation can be broadly categorized into three distinct field complexity theory, computability theory and automata theory. The study opts for a secondary approach that involves already existing resources to explore, understand and enhance effectiveness in the research. The theory of computer expertise and complexity expects a well-defined description of a computer automata theory that provides exercise with the formal design of discretion. It acquaints theories applicable to other known non-theoretical spheres of computer science. The study emphasizes on comprehensively analyzing the theory of computation and its key consideration, along with trying to determine the proximity in resolving the problem.
\end{abstract}

Keywords: Theory of Computation, complexity theory, automation theory and computability theory

\section{Introduction}

The traditional principles of computation prospered as an autonomous systematic discipline due to the zenith of concepts from all relevant and diverse professions in science and technology. The computational theory transpired through an accelerating development phase from 1970 to the 1990 s and has encountered critical maturity considering then. The enormous prominence of computer and information technology at the commencement of the $21 \mathrm{st}$ century has sincerely valued an academic experience to acquire on the matter, which originally perceived all viewpoints of our social, technological, and experimental lives [1]. The theory of computation is an innovative, significant and fascinating theory of mathematics, physics, and other frequently addressed themes.

Computers and related technology have previously authenticated themselves as an indispensable mathematical exercise with improving associations in mathematical spheres. Text developing connection and synergy with all sciences, naturally integrative computational design algorithms, and complexity in couple assumptions of nature and civil labels the inception of another logical revolution[2]. Theory of computation is essentially inspired by a necessity for consideration and modelling emanating from the different intersecting system, ordinarily carrying appearance to a novel domain of research as a theoretical basis of computation and complexity, analytical model of intelligent, theoretical computation paradigm of parallel computation, and a study of obstinacy and inapproximability encouraged by scientific optimisation. Artificial intelligence is a description of the twenty-first century with reliable seams to the theory of computation; remarkable of them are machine learning interpretation and conception of intelligent or evolutionary algorithm performance and critique of trend information and adequate algorithm in data science and big data technology.

The purpose of the theory of computation evolution is to emerge formal mathematical principles of computation that indicate real-world machines. The theory of computation can be broadly categorized into three distinct field complexity theory, computability theory and automata theory.

\section{1) Complexity Theory}

Computational complexity is a branch of the theory of computation which is executed to interpret how troublesome an obstacle is resolved and the standard means time and space determined and appropriated during the execution of the barrier. The kinds of time complexity usually involve $\mathrm{P}$, NP, NP-hard, NP-complete, and others; space complexity groups generally comprise PSPACE, NPSPACE, PSPACEhard and PSPACE-complete. Studying the computational complexity of a problem can determine whether there is an adequate solving algorithm for the situation or not. The noticeable question in complexity method associated like analyze problems according to their level of hardness.

Complexity theory essentially concentrates on the worstcase complexity of computational difficulties. It is a subsidiary of the theory of computation. Theoretical computer science directs on organizing and computational problems based on their intrinsic test and associated properties [3]. A computational problem is perceived to be a job that is in teaching tractable to remaining determined by a computer which is comparable to affirming that the issue may be resolved by an automatic application or analytical measures such as an algorithm. More specifically, computational complexity theory attempts to distinguish problems that can or cannot be resolved with properlyregarded support.

\section{2) Computability Theory}

Computability theory, also recognized as recursion theory, is a category of mathematical reasoning of computer science and the theory of computation that began in 1930 with calculable capacity and Turing degree [3]. It is a field that extends to numerous dimensions, comprising the research of generalized computability and definability. However, it is an analysis that recursion theory overhangs with the case theory and functional graphic set theory.

The noticeable question approached by recursive theory involving

a) What does it suggest for a purpose on the natural number to be estimable? 
b) How can non-computable purposes be divided into a theocracy based on their strata of non-computability?

c) Identified the problem as solvable and unsolvable?

The theory of computation technique and complexity is nearly compared to each other. In complexity theory, the purpose is to analyze problems as simple ones and difficult ones. In contrast, in computability theory, the distribution of the issues is by those that are discernible. Those that are not computability principles include numerous of the approaches used in complexity theory.

\section{3) Automation Theory}

Automation theory deals with the determination and features of mathematical patterns of computation. These patterns perform a vital role in several connected regions of computer science [4]. Automata theory is a matter of complex machines and automata as well as computational problems that can be determined employing them. It is an assumption in academic computer science and discrete mathematics.

The term automata indicates self-acting. One of the principles called finite automation is practised in text processing, compilers and hardware designing, another type, called context-free grammar, is practised in programming languages and artificial intelligence. Automata theory is an attractive position of continuing the building of the study of the theory of computation. The theory of computer expertise and complexity expects a well-defined description of a computer automata theory that provides exercise with the formal design of discretion. It acquaints theories applicable to other known non-theoretical spheres of computer science.

Automation theory is closely related to symmetrical language theory, and automation is a terminable description of a standard language that may be an absolute set. The traditional language group often incorporates automation; they can ascertain size typically explained by the Chomsky hierarchy, which defines the connection between multiple languages and formalized reasoning. Automata perform a significant purpose in the theory of computation, compiler architecture, Artificial intelligence boosting and formal affirmation.

\section{Research Objective}

The paper's motive is to comprehensively analyze the theory of computation and its key consideration, along with trying to determine the proximity in resolving the problem.

\section{Research Question}

Analysis of the theory of computation and key considerations of computational problems?

\section{Literature Review}

The papers illustrate an introduction of theory by discussing elements and significant features, and details of the theory of computation. The theory of computation is a section of mathematics and computer science. An enormous number of principles which are addressing computation is implemented in computer hardware and software[1]. The theory of computation can be suitably organized into three all- inclusive classifications: complexity theory, computability theory, and automata theory. The motive of the study is a too extensive analysis of the theory of computation how a mathematical medium efficiently employed by performing certain principles, patterns, models of computation, and the associated semantics from the most foundational to the vaguest like finite automata and formal language, contextfree language, and pushdown automata, and the turing machine, and recursively enumerable and recursive language[3]. It also explores decision problems to reduce and undecidability on the Kleene program to computability, complexity, and NP-completeness. The persistence of the theory of computation progression is to emerge formal mathematical principles of computation that indicate realworld machines [4]. The computational principles demonstrate significant characteristics categorized in three distinct fields: complexity theory, computability theory, and automata theory which also encompasses turning machines, computational complexities, and many others [6]. Computers are performing an indispensable role as language acceptors. Acquiring language is relatively similar to resolving a decision problem. Many fascinating computation intricacies can be functioning as decision problems; certain designs exclusively meant for computation intelligence of delivering the likewise consequences more intricate than yes and no [7]. Theory of computation is truly stimulated by a compulsion for evidence and modeling originating from the diverse intersecting practice, frequently moving appearance to a novel specialty of research as a logical basis of computation and complexity, analytical model of intelligent, theoretical computation paradigm of parallel computation, and a study of obstinacy and unpredictability inspired by scientific optimization [8]. The teaching and learning theory of computation is a challenging responsibility from both perspectives since it concerns the conceptual proposal and mathematical experience [6]. Additionally, the description of its significance to other computer science programs is inapplicable. Due to the sense of closed cooperation among intellectual and practiced science, impulse among learners is expected, which may develop an interest in computationrelated theories and viewpoints. The theory of computation technique and complexity has approximately corresponded to each other. In complexity theory, the objective is to analyze intricacies as simple ones and difficult ones [2]. In contrast, in computability theory, the distribution of the concerns is by those that are discernible. Those that are not computability principles include numerous of the approaches used in complexity theory. The paper's motive is to introduce a distinct methodology for making interactive and realistic measures to enhance teaching and learning based on the theory of computation [8]. The paper involves teaching methodology, including the introduction of computers in programming data structure NLP and compilers with automatic course enriching automata course by the inclusion of problems as well as natural language processing [10]. Formal language theory and the theory of computation is notably difficult as it immensely entails mathematical stuff for computer science. Ordinarily, the mathematical material dampens learners and generates enormous hurdles. The paper [11] highlights prominent discovery learning techniques known as the Moore method, which stimulate the excitement, curiosity, and discipline for learning the theory of computation. Another search [13] 
implements theory of computers to explain the architectural, thermodynamics, automated, and optical qualities of Nanoscale material. Nanoscience illustrates the dynamic potential and outstanding opportunity for theory and computation to strive way in the innovation manner because the experimental to present an inadequate understanding of the structure of nanomaterial and theory can satisfy these loopholes and excerpt features crucially by the theory of computation to simulate exploring and learning to understand the topic.

\section{Method}

The study opts for a secondary approach that involves already existing resources to explore, understand and enhance effectiveness in the research. It utilizes online and offline resources like research papers, literature, public reports, international organization reports and other internet website data relevant to the research. In this research, more than 50 research papers were initially selected to understand the objective of the research thoroughly. Subsequently, for research writing, the study finally selected 15 research papers and relevant internet resources for obtaining appropriate finding and conclusion.

\section{Discussion}

Real-world computers implement a computation that, by variety like mathematical principles, determines queries systematically. The structure of the theory of computation is to serve in advancing analytical and rational principles that efficiently and to halt. Overall, the mechanisms that execute reasoning employ computational learning theory, theory of computation to provide scholars an insight into computer hardware and software constraints. The significance of analyzing computation is to experience better-

- The advancement of formal mathematical patterns of computation indicates the real world of computers.

- To revise the constraints of computers and clarify what classification of queries can be computed, the particular purposes of the theory of computation are essential.

- Formulating effective algorithms that operate on computer devices.

- Programming semantics analysis and their evolution.

- Effective compiler configuration and installation.

The key consideration of computation problem is-

- Whatever can and cannot be measured

- The activity of such computation

- The significance of memory is practiced during exploration computations.

The computable and uncomputable problem in the theory of computation

The purposes and the computable queries mean there endures some algorithm that computes can be accountable, any instance of the problem for any facts, information to be effectively functioning in a measurable number of manageable moves. Some of the prominent example of computation problem such as- a) Computing the largest common divisor of a couple of integrals.

b) Arrange the shortest path between a combination of links in a finite graph.

Although some specific queries and functionalities are uncomputable, no algorithm can estimate or produce the result for all the appropriate facts in a finite capacity of manageable moves. That implies no algorithm has the capability to determine the relevant problem. One such example is the halting problem.

\section{Conclusion}

Theory of computation is essentially inspired by a necessity for consideration and modeling emanating from the different intersecting system, ordinarily carrying appearance to a novel domain of research as a theoretical basis of computation and complexity, analytical model of intelligent, theoretical computation paradigm of parallel computation, and a study of obstinacy and inapproximability encouraged by scientific optimization. Theory of computation is truly stimulated by a compulsion for evidence and modeling originating from the diverse intersecting practice, frequently moving appearance to a novel specialty of research as a logical basis of computation and complexity, analytical model of intelligent, theoretical computation paradigm of parallel computation, and a study of obstinacy and unpredictability inspired by scientific optimization.

Eventually, the theory of computation acts as a bedrock for cutting-edge technology like artificial intelligence, big data, and IoT, among others. Effective and adequate algorithms lead to better programs that optimally use hardware resources. Good understanding of the theory of computation of system programmers and developers express themselves clearly and intuitively.

\section{References}

[1] Daneshgar A. Theory of Computation;2020.

[2] Maheshwari A, Smid M. Introduction to theory of computation. InSchool of Computer Science Carleton University Ottawa Canada 2014 Sep 25.

[3] Sharma M, Chaudhary N, Khubchandani S. An Introduction of Theory of Computation.

[4] Sipser M. Introduction to the Theory of Computation. Cengage learning; 2012 Jun 27.

[5] Lewis HR, Papadimitriou CH. Elements of the Theory of Computation. ACM SIGACT News. 1998 Sep 1;29(3):62-78.

[6] Goyal M, Sachdeva S. Enhancing theory of computation teaching through integration with other courses. International Journal of Recent Trends in Engineering. 2009 May 1;1(2):137.

[7] Back K. A course in derivative securities: Introduction to theory and computation. Springer Science \& Business Media; 2006 Mar 30.

[8] Martin JC. Introduction to Languages and the Theory of Computation. NY: McGraw-Hill; 1991.

[9] Core A. Theory of Computation.

[10] Sigman S. Engaging students in formal language theory and theory of computation. InProceedings of the 
38th SIGCSE technical symposium on Computer science education 2007 Mar 7 (pp. 450-453).

[11] Smith C. A recursive introduction to the theory of computation. Springer Science \& Business Media; 2012 Dec 6.

[12] Lein M. Characterization of agostic interactions in theory and computation. Coordination Chemistry Reviews. 2009 Mar 1;253(5-6):625-34.

[13] Schatz GC. Using theory and computation to model nanoscale properties. Proceedings of the National Academy of Sciences. 2007 Apr 24;104(17):6885-92.

[14] Martin JC, Blanco y Correa Magallanes JL. Introduction to languages and the theory of computation. Lenguajes formales y teoría de la computación. 2004.

[15] Vijayalakshmi M, Karibasappa KG. Activity based teaching learning in formal languages and automata theory-An experience. In2012 IEEE International Conference on Engineering Education: Innovative Practices and Future Trends (AICERA) 2012 Jul 19 (pp. 1-5). IEEE.

\section{Links}

[16] https://www.section.io/engineeringeducation/introduction-to-theory-of-computation/

[17] https://www.geeksforgeeks.org/computable-and-noncomputable-problems-in-toc/ 